\title{
Numerical study of viscous modes in a rotating spheroid
}

\author{
D. Schmitt \\ LGIT, CNRS-UJF, BP 53, 38041 Grenoble Cédex 9, France
}

\begin{abstract}
The motion of an incompressible, viscous rotating fluid contained in an oblate spheroidal container is studied by a direct numerical simulation in an appropriate spheroidal coordinate system and in the linear approximation. The behaviour of a few eigenmodes is investigated as a function of the eccentricity $e$ of the container, for a Ekman number $E=10^{-5}$. Viscous effects are evidenced through internal shear layers, the spatial structure of which strongly depends on the eccentricity. In particular, for the spin-over mode, a resonance occurs around a critical value $e_{c} \approx 0.50$, where the decay rate strongly deviates from the predicted theoretical variation. This resonance is discussed in relation with the accidental coincidence between the spin-over frequency and two other frequencies corresponding to the $(8,1,5)$ and $(14,1,9)$ inertial eigenmodes.
\end{abstract}

Keywords : Rotating flows, Navier-Stokes equations, Waves in rotating fluids.

\section{Corresponding author :}

SCHMITT Denys

LGIT - CNRS-UJF

BP 53, 38041 Grenoble Cedex 9, FRANCE

Tél : (33) 4.76.82.80.28

Fax : (33) 4.76.82.81.01

email : Denys.Schmitt@obs.ujf-grenoble.fr 


\section{Introduction}

Understanding the dynamics of rotating fluids is of fundamental interest in a geophysical and astrophysical point of view. As far as the geodynamo problem is concerned, the behaviour of inertial modes of the liquid inner core of the Earth in the presence of external constraints such as the ellipticity of its outer boundary and the precession forcing appears worth being carefully investigated. The spheroidal geometry of this outer boundary was explicitely considered by Poincaré (Poincaré, 1910), giving rise to specific effects on the Earth's nutation compared to the case of a spherical container, but viscosity was neglected. Viscosity was later considered as a perturbation of the non viscous modes, with a special attention to the critical regions of the Ekman layer (Busse, 1968; Greenspan, 1968; Stewartson \& Roberts, 1963). In particular, viscous correction of decay rate and eigenfrequency for the spin-over mode was given analytically as a function of the ellipticity of the container. This was recently generalized for any inertial wave mode (Liao, Zhang \& Earnshaw, 2001; Zhang, Liao \& Earnshaw, 2004).

Following these fundamental analytical approaches, numerical studies have been undertaken in a sphere or a spherical shell (Hollerbach \& Kerswell, 1995; Noir, Jault \& Cardin, 2001; Rieutord \& Valdaretto, 1997; Tilgner \& Busse, 2001). Indeed, such simulations are needed to go further into our knowledge of the phenomena and to explain experimental results where fluid instabilities and turbulence are observed and reveal non-linear effects. In particular, inertial modes are suspected to play a role in the geodynamo, because some of them could be destabilized by the elliptic shape of the boundaries due to the tides (Kerswell, 1994).

Most of the numerical studies of rotating fluids have been developed in a spherical geometry, because of its relative simplicity. Using a more realistic coordinate system appears however highly desirable, because natural bodies or cavities are often 
ellipsoidal rather than spherical. Such an approach has been used for studying kinematic dynamo models in a spheroidal galaxy (Walker \& Barenghi, 1994). More recently, a spheroidal symmetry has been considered for some geophysical applications (Lorenzani \& Tilgner, 2001; 2003; Tilgner, 1999). In the latter approach, a coordinate transformation was performed in order to replace the ellipsoidal volume by a spherical one, for which a distorted equation of motion was used. As well, a solid inner core was included, allowing to avoid singularities at the centre.

In the present work, equation of motion of a rotating viscous incompressible fluid within a spheroidal container is directly treated in real space by using an appropriate set of coordinates, namely the oblate spheroidal coordinate system. Within this system, an extension of the spherical Mie representation of a vector field (Backus, Parker \& Constable, 1996) is considered: a solenoidal vector field (here the velocity field) is decomposed into the sum of two unique vectors which are themselves derived from two scalar fields, namely the sphero-poloidal and the sphero-toroidal ones. As for the spherical symmetry, this Mie-like representation allows one to work with only two scalar fields instead of the three velocity components, once the pressure has been eliminated from the equations. In addition, an appropriate spherical harmonic expansion is used for the angular part of these spheroidal scalar fields, while a discretisation is performed for their sphero-radial component. The main features of this formalism have been previously presented in detail, but only a few preliminary numerical results were included (Schmitt \& Jault, 2004). Here, numerical simulations are performed more thoroughly. In particular, the behaviour of a few viscous eigenmodes is investigated as a function of eccentricity and, to a lesser extent, of viscosity. As well their spatial geometry is emphasized, exhibiting features which suggest the presence of possible resonance between them, under certain circumstances.

In the present work, the linear approximation is made, and no inner core is considered. In Section 2, the mathematical formalism within the oblate spheroidal 
coordinate system is briefly recalled, and two distinct procedures are described to solve the equation of motion, namely a time-stepping and an inverse iteration method. Section 3 is devoted to the numerical results of a systematic study of a particular mode, i.e. the spin-over mode, as a function of the excentricity and for a Ekman number $E=10^{-5}$, showing the presence of a resonance around a critical eccentricity $e_{c}=0.50$. This behaviour differs markedly from what was expected in previous theoretical approaches. In the next Section, the behaviour of the other spheroidal, viscous eigenmodes is considered and their role in the occurence of the spin-over resonance is emphasized. The last Section is devoted to the discussion.

\section{Mathematical formalism}

\subsection{The oblate spheroidal coordinate system}

A coordinate system particularly appropriate to the present study (spheroidal container, no inner core) is the oblate spheroidal system, related to the cartesian one by the relations

$$
\begin{aligned}
& \square x=a \cosh \square \sin \square \cos \square \\
& y=a \cosh \square \sin \square \sin \square \\
& z=a \sinh \square \cos \square
\end{aligned} \quad \text { with } \begin{aligned}
& \square \square \geq 0 \\
& 0 \square \square \square \square \\
& 0 \square \square<2 \square
\end{aligned}
$$

where $a$ is the distance between the origin and the circle of foci within the equatorial plane. Within this system, the constant- $\square$ surfaces are ellipsoids with eccentricity $e=1 / \cosh \square$, while the constant- $\square$ surfaces are one-sheet hyperboloids which, for large $\square$ values, become asymptotically cones of revolution around the $z$-axis with a halfaperture $\square$. Details of the formalism can be found in (Schmitt \& Jault, 2004), and only a summary is given here below, including some changes compared to the previous work.

\subsection{Decomposition of scalar and vectorial fields in the spheroidal symmetry}

As shown by (Schmitt \& Jault, 2004), an appropriate transformation allows one to expand any scalar function $\mathrm{f}(\square, \square, \square)$ within the usual spherical harmonics basis 
$Y_{l}^{m}(\square, \square)$, where $\square$ is the spheroidal, not the spherical colatitude. In the same way, a solenoidal vector field $\boldsymbol{V}(\square, \square, \square)$ can be decomposed into the sum of a sphero-toroidal $\boldsymbol{V}_{s t}$ and a sphero-poloidal $\boldsymbol{V}_{s p}$ part, with:

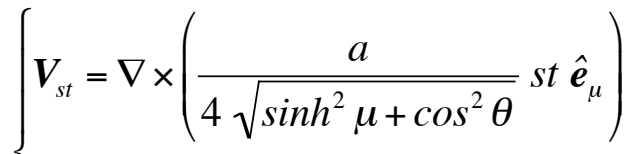

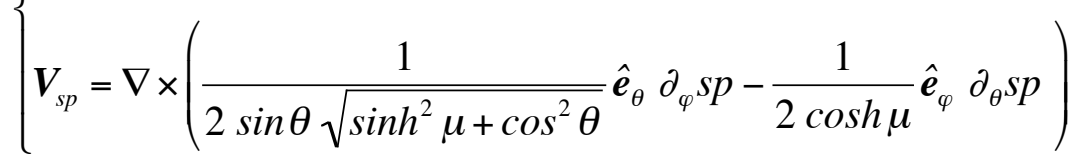

where $\partial_{\square}$ is a short notation for $\partial / \partial_{\square}$, and $\operatorname{st}(\square, \square, \square)$ and $\operatorname{sp}(\square, \square, \square)$ are the spherotoroidal and sphero-poloidal scalar fields of $\boldsymbol{V}$, respectively. This decomposition ensures the vanishing of $\square . V$, in agreement with the incompressibility of the fluid. Note that differences exist compared to the Mie decomposition in spherical symmetry, for example the curl of a sphero-poloidal vectorial field is not a sphero-toroidal field, nor the opposite assertion.

\subsection{Equation of motion}

The present work is focused on the study of viscous correction to inviscid eigenmodes in the linear approximation. An incompressible viscous fluid is enclosed in an oblate spheroidal container of sphero-radial coordinate $\square_{o}$ and eccentricity $e_{o}=1 / \cosh \square_{o}$. The container is spinning at frequency $\square_{o}$ around the $z$ axis. The linear, dimensionless equation of motion for the fluid velocity $\boldsymbol{u}$ within the coordinate system rotating with the container is:

$$
\partial_{t} \boldsymbol{u}+2 \hat{z} \square \boldsymbol{u}=\square \square \square+\frac{E}{e_{o}^{2}} \square \boldsymbol{u}
$$

where $\square$ is the reduced pressure and $E$ the Ekman number. Here the focus parameter $a$ has been taken as unit of length, and $\square_{o}^{-1}$ as unit of time.

After eliminating the pressure by taking the curl of equation (2.3), three coupled, differential equations can be obtained for the two unknown quantities $\operatorname{st}(\square, \square, \square)$ and $s p(\square, \square, \square)$ after projection on the three basis vectors $\hat{\boldsymbol{e}}_{\square}, \hat{\boldsymbol{e}}_{\square}, \hat{\boldsymbol{e}}_{\square}$. Note that these three 
equations are not independant, and this will be used here below to reduce the order of $\square$ derivatives. These equations involve $\square$-derivatives up to the fourth-order, and $\square$ and $\square$ derivatives up to the fifth order. It is worth noting that the $\square$-derivation is straightforward when the spherical harmonic expansion is considered, while the order of the $\square$-derivatives can be reduced down to first order by using the angular laplacian operator. After some manipulation, the $\square$-dependence includes numerous terms such as $\cos ^{n} \Pi$ or $\cos ^{n \square 1} \square \sin \square \partial_{\square}$, where $n$ can be as large as 7 , but no high-order $\square$-derivative. As far as $\square$-derivatives are concerned, a careful examination of the boundary conditions (see below section 2.4) induces one to keep at most fourth-order derivatives for $\operatorname{sp}(\square, \square$ $\square)$ and second-order derivatives for $\operatorname{st}(\square, \square, \square)$, a condition which is fulfilled for the $\square-$ equation. However, both $\square$ - and $\square$-equations involving $\partial_{\square s t}^{3}(\square, \square \square)$, an appropriate combination of them has been considered in order this term to be cancelled. For sake of conciseness, the two remaining $\square$ - and $(\square \square)$-equations are not given here explicitely.

The next step is to use the spherical harmonic expansion for both scalar fields $s t(\square, \square, \square)$ and $s p(\square, \square, \square)$. Thus, the $\square$-dependent operators quoted above, e.g. $\cos ^{n} \Pi$, when applied to a function $Y_{l}^{m}$, can be expanded into the sum of $(n+1)$ spherical harmonics with the same order $m$, and degrees of same parity ranging from $l-n$ to $l+n$. The system of equations can then be reduced to expressions which involve partial derivatives in $\square$ and $t$ only, and are expanded in spherical harmonics. Finally, considering separately each given $l$ angular part provides a set of coupled equations which include numerous contributions coming from terms with other angular parts up to $\square l=7$ (i.e. from $l-7$ to $l+7$ ). All the expressions have been derived by using Mathematica software.

\subsection{Boundary conditions}

The no-slip condition has been used at the outer boundary of the container, i.e. $\boldsymbol{u}\left(\square_{o}\right)=$ 0 . This condition leads to the following relations: 


$$
s t_{l}^{m}\left(\square_{o}\right)=0 \quad ; \quad s p_{l}^{m}\left(\square_{o}\right)=0 \quad ; \quad \partial_{\square} s p_{l}^{m}\left(\square_{o}\right)=0
$$

where $s p_{l}^{m}(\square)$ and $s t_{l}^{m}(\square)$ are the spherical harmonic expansion coefficients of the $s p$ and $s t$ scalar fields. A second set of relations comes from the critical disk $\square=0$ enclosed of the focal circle. The absence of inner core implies that $\boldsymbol{u}$ and its derivatives are continuous when passing through this surface, both sides of this surface corresponding to $\square$ and $\square-\square$. That leads to the following parity relations for the functions $s t_{l}^{m}(\square)$ and $s p_{l}^{m}(\square)$ for small values of $\square: s p_{l}^{m}(\square)$ and $s t_{l}^{m}(\square)$ must be odd if $(l+m)$ is even, and even if $(l+m)$ is odd. In the present calculation, this is equivalent to the vanishing of $s t_{2 n+1}^{1}(0), \partial_{\square} s p_{2 n}^{1}(0), \partial_{\square}^{3} s p_{2 n}^{1}(0)$. Note that the choice of the latter term rather than $\partial_{\square}^{2} s t_{2 n+1}^{1}(0)$ lies on the following argument: if an inner core was used, the boundary condition would be the same as equation (2.4), but for $\square=\square_{i}$, i.e. one condition on $s t_{l}^{m}\left(\square_{i}\right)$ and two on $s p_{l}^{m}\left(\square_{i}\right)$. Note that this choice remains consistent with the order of the derivatives of $s t_{l}^{m}(\square)$ and $s p_{l}^{m}(\square)$ in the equation of motion, i.e. 2 and 4 , respectively.

\subsection{Numerical methods}

Numerical calculation is made by using the finite difference method for the sphero-radial variable $\square$. The grid is linear, except near the boundaries where it follows a geometric law: the grid is then extended near the critical disk $\square=0$ in order to reduce numerical problems near the focal circle, while it is compressed close to $\square=\square_{o}$ in order to increase the number of points within the Ekman boundary layer. The total number of points is generally taken as $N=251$. For the angular variables, the spherical harmonic expansion is truncated at $\operatorname{lmax}=64$. For the azimutal part, only $(m=1)$ terms are needed here for the spin-over problem (see section 2.6), the $(m=-1)$ terms being related to the $(m=1)$ one through complex conjugation.

The corresponding matrices consist in a block-pentadiagonal matrix associated with the radial grid, each block being a band-matrix of order lmax, arising from the coupling of scalar field components having different $l$ values. Moreover, due to symmetry considerations, two independent groups of these components can be 
considered separately: the first group involves $s p_{1}^{m}, s t_{2}^{m}, s p_{3}^{m}, s t_{4}^{m} \cdots$, while the second one, which is used here below, includes $s t_{1}^{m}, s p_{2}^{m}, s t_{3}^{m}, s p_{4}^{m} \cdots$.

As far as the time dependence is concerned, two different method have been considered. First, the implicit Crank-Nicolson scheme has been used for the diffusion term (initial value problem). For each time step, the inversion of the big pentadiagonal matrix is carried out by LU factorization and Thomas algorithm, in order to avoid keeping in memory the inverse of this matrix. The LAPACK routines have been used for manipulating the matrices. In the second procedure the inverse iteration method has been applied (Stoer \& Bulirsch, 1980), which provides complex eigenvalues, i.e. both eigenfrequency and attenuation rate, as well as eigenvectors. The selection of the final eigenmode is made by starting the iterations from an initial complex eigenvalue close to the expected value. Any individual, viscous eigenmode can then be achieved by this procedure, but identifying the corresponding $(n, m, k)$ labels may not be trivial in particular for not small values of $n$. Moreover, the final eigenmode is generally not very sensitive to the imaginary part of the starting value, i.e. the initial attenuation rate, except when eigenvalues are close to each other. That may explain the presence of some irregularities on the calculated variations (see below). In the following, both methods have been used to study the spin-over behaviour, while other eigenmodes have been investigated only by the inverse iteration method.

\subsection{Description of the initial inviscid spin-over mode}

In the Crank-Nicolson scheme, an initial condition has to be taken as the starting $(t=0)$ velocity field. For the spin-over investigation, the choice is the $(2,1,1)$ inviscid inertial (Poincaré) mode, for which all the components are purely linear in the spatial coordinates $(x, y, z)$ and which satisfies the non-penetration condition on the spheroidal container boundary $\square=\square_{o}($ Greenspan, 1968; Kerswell, 1993):

$$
\boldsymbol{u}_{211}=\frac{z}{\tanh \left(\square_{o}\right)} \hat{\boldsymbol{e}}_{y} \square \tanh \left(\square_{o}\right) y \hat{\boldsymbol{e}}_{z}
$$


This mode corresponds to a vortical flow around the $\hat{\boldsymbol{e}}_{x}$ axis, with a constant-vorticity and no boundary layer. This inviscid inertial mode is excited when a fluid is in rapid rotation around the $z$-axis and its rotation axis is suddenly tilted by a slight quantity (Greenspan, 1968). Precession may be viewed as a sequence of such infinitesimal changes so that it is anticipated that this mode plays a key role in that problem. Writing equation (2.5) in the spheroidal coordinate system and expanding the corresponding scalar fields in spherical harmonics lead to only three non-zero terms which involve only $m=1$ (see (Schmitt \& Jault, 2004)), namely $s t_{1}, s p_{2}, s t_{3}$ (here and in the following, the index $m=1$ has been omitted for sake of simplicity). It is worth noting that the boundary conditions are verified at $\square=0$ but not at $\square=\square_{c}$ for these three scalar fields. The values at $\square_{\square}=\square_{c}$ for $s t_{1}$ and $s t_{3}$, as well as the previous one at $\square_{\square \square 1}$ for $s p_{2}$ need to be modified in order to fulfill the outer boundary conditions and to take into account the viscosity through equation (2.4). As it will be seen below, a consequence of this abrupt change is to excite high-order viscous eigenmodes which will be visible in the time dependences shown below, giving rise to rapidly decaying transient oscillations.

\subsection{The angular momentum}

The expression of true vorticity in the spheroidal system being complex to evaluate, an alternative, more simple way to follow the evolution of the rotating fluid is to calculate its angular momentum $\ell$, defined as (the prefactor has been removed):

$$
\ell=\underset{\text { spheroid }}{\square} \square \boldsymbol{u}
$$

Within the spherical limit, this vector is nothing else than half of the true vorticity, $(1 / 2) \square \square \boldsymbol{u}$. In spheroidal symmetry, expanding the scalar fields of the velocity field $\boldsymbol{u}$ in spherical harmonics and integrating their angular parts leads to an expression which involve only $s t_{1}$ and $s p_{2}$. Moreover, it turns out that this angular momentum, apart a constant prefactor, is nothing else but the projection of the vector field $\boldsymbol{u}$ on the two 
orthogonal inviscid spin-over modes $\boldsymbol{u}_{211}$ and $\boldsymbol{u}_{2 \overline{1} 1 \text { which correspond to the inertial }}$ vortical flows around $\hat{\boldsymbol{e}}_{x}$ and $\hat{\boldsymbol{e}}_{y}$, respectively:

$$
\left.\left.\ell=\square \operatorname{coth}\left(2 \square_{c}\right) \prod_{\text {pheroid }}^{\square} \square_{211}{ }^{*} \cdot \boldsymbol{u}\right) \hat{\boldsymbol{e}}_{x}+\square_{\text {spheroid }} \boldsymbol{u}_{2 \overline{1}}{ }^{*} \cdot \boldsymbol{u}\right) \hat{\boldsymbol{e}}_{y} \underline{E}
$$

The evolution of the system will then be followed by investigating the time-variation of this vector $\ell$, which remains confined within the equatorial plane, as well as its dependence on viscosity and eccentricity.

\section{Numerical results}

\subsection{Time dependence of decay factor and eigenfrequency}

The angular momentum $\ell$ can be defined by its modulus $l_{\text {mod }}$ and its position. From a theoretical point of view, $l_{\text {mod }}$ is expected to decay exponentially with time according to viscous effects (negative decay factor $\square$ ), while the vector $\ell$ is expected to rotate in the equatorial plane in a retrograde way (negative angular speed $\square_{d}$ ):

$$
\begin{aligned}
& \square_{\text {mod }}=l_{0} \exp (\square t) \\
& \square\left(\ell, \hat{\boldsymbol{e}}_{x}\right)=\square+\square_{d} t
\end{aligned}
$$

where $\left(\ell, \hat{\boldsymbol{e}}_{x}\right)$ is the angle between vectors $\ell$ and $\hat{\boldsymbol{e}}_{x}$. At each time step, the decay factor is then calculated as $\mathrm{d}\left(l_{\text {mod }}\right) / l_{\text {mod }}$ and the angular speed as $\mathrm{d}\left(\ell, \hat{\boldsymbol{e}}_{x}\right) / \mathrm{d} t$. Two typical time dependences of both quantities are reported in figure 1 for a Ekman number $E=10^{-5}$. That allows one to appreciate the time-stepping procedure as well as the effects of ellipticity. After some long period transient oscillations, a stationary state is reached for large $t$ values, where residual small oscillations subsist around a constant value for both decay rate $\square$ and eigenfrequency $\square_{d}$.

\subsection{Eccentricity dependence of decay factor and eigenfrequency}


The eccentricity dependence of the large $t$ limit of decay rate and eigenfrequency is reported in figure 2 , together with the values calculated directly by the inverse iteration method. Both results are in perfect agreement between each other, proving that the former ones are not very affected by the vanishing residual modes which still subsist in the calculation for large $t$. In other words, the large $t$ limit is very close to the pure viscous spin-over eigenmode. These variations can be compared to those expected from a theoretical point of view according to the following relations (Greenspan, 1968; Stewartson \& Roberts, 1963; Zhang et al., 2004):

$$
\begin{aligned}
& \square=\square_{l}(e) E^{1 / 2}+O\left(E, e^{4} E^{1 / 2}\right) \\
& \square_{d}=\square_{0}(e)+\square_{l}(e) E^{1 / 2}+\ldots \\
& \text { with } \square_{1}(e)=\square 2.62 \square 0.426 e^{2}, \\
& \square_{0}(e)=\frac{\square 2}{2 \square e^{2}} \text { and } \square_{1}(e)=\square 0.258 \square 0.766 e^{2}
\end{aligned}
$$

The renormalized decay rates as well as the eigenfrequencies are found to satisfactorily follow the predicted variation, at least for moderate eccentricities. The $\square_{1}$ variation is a little more erratic, but it is worth noting that this quantity is a high order one in the expansion of $\square_{d}$, therefore it is subject to a lower degree of accuracy. However, a spectacular change of behaviour clearly occurs around $e_{c} \approx 0.5$, emphasized by the strong deviation of $\square$ from the $e^{2}$ dependence. Unfortunately, the appearance of numerical instabilities for $e>0.6-0.7$ prevents us to analyze the behaviour for higher eccentricities. In order to have a deeper understanding of what happens around this critical eccentricity, the spatial structure of the spin-over mode has to be examined carefully in the presence of both viscosity and eccentricity.

\subsection{Spatial structure of the spin-over mode}

The detailed spatial structure of the flow resulting from the combined effect of the viscosity and the non-spherical shape of the container can be appreciated by subtracting an appropriate non-viscous spin-over contribution from the calculated viscous flow. The 
three components of the residual fluid velocity are then visualized within the meridional plane containing the rotation axis of the removed spin-over contribution or equivalently the $\ell$ vector itself (see figure 3 ). Note that the spatial structures calculated by both methods are undistinguishable from each other. They exhibit shear regions, although the relatively high Ekman number limits the narrowness of the shear layers. The main characteristic feature is the spatial evolution of the azimutal component $u_{\square}$. While for $e$ $=0.02$, features are similar to those calculated by (Hollerbach \& Kerswell, 1995) and (Noir et al., 2001) in spherical geometry, the $u_{\square}$ component exhibits more pronounced cells when $e$ increases, in a way reminiscent of some inviscid spheroidal inertial modes (see below). Nevertheless these structures are actually characteristic of the viscous spin-over eigenmode $(2,1,1)$, so that explaining the presence of such features requires to investigate in more detail the behaviour of the spheroidal inertial eigenmodes themselves and their dependence on viscosity and eccentricity.

\section{The spheroidal inertial eigenmodes}

\subsection{The non-viscous inertial modes}

First we focus on the non-viscous spheroidal inertial modes, which are plane waves solutions of equation 2.3 for $E=0$. According to the notation of Kerswell (Kerswell, 1993), there are an infinite number of such eigenfunctions $Q_{n, m, k}$ (the Poincaré modes), depending on the three labels $n, m, k$ :

$$
Q_{n, m, k}(\square, \square, \square, t)=Q_{n, m, k}(\square, \square) e^{i\left(m \square+\square_{n, m, k} t\right)}
$$

where $n$ and $m$ refer to the associated Legendre polynomials $P_{n}^{m}$ which are involved in the analytical expression of the eigenmodes and eigenfrequencies, while $k$ subscript labels the different eigenfrequencies $\square_{n, m, k}$ corresponding to a given set of $(n, m)$ values. These frequencies depend on the eccentricity $e$, as shown in figure $4 \mathrm{a}$ for a selection of eigenmodes, the frequency of which is close to that of the spin-over one, $\square_{2,1,1}$ (note that 
this latter is nothing else but $\square_{o}$ of equation 3.2, except the sign), i.e. close to 1 . Clearly the $(8,1,5)$ and $(14,1,9)$ modes are expected to play a particular role, and it is worth considering their spatial distribution (see figure 5 for $e=0.39$ for example). Note that their global geometry is not drastically modified between the spherical case, $e=0$, and a noticeable oblate case, $e=0.7$. While the non-viscous $(2,1,1)$ mode always exhibits a very simple laminar structure - it looks like a stretched solid body rotation -, it immediately appears that the geometrical features of both $(8,1,5)$ and $(14,1,9)$ modes are very close to those present in the calculated viscous spin-over mode (see section 3.3). They are even both present in a single viscous eigenmode, if the azimutal variation of the three components is considered (see figure 6 for $e=0.39$ ). Therefore, it can be anticipated that a viscosity induced resonance occurs between all three inertial modes, in the range of eccentricity where there is a crossing of their frequencies. So it is interesting to investigate more thoroughly how the different inertial modes are affected by the viscosity.

\subsection{The viscous inertial modes}

Including viscosity in equation 2.3 prevents us to solve analytically the eigenproblem. In contrast, the inverse iteration method may allow to obtain viscous solutions for different modes and to appreciate how their eigenfrequency, decay rate and spatial structure are modified by the viscosity as a function of eccentricity. As seen in figure 4a, the viscous eigenfrequencies agree generally well with the corresponding inviscid variation. Nevertheless, difficulties may sometimes occur to follow some modes over the whole eccentricity range, as for example the $(14,1,9)$ mode for $e>0.4$ or the $(20,1,13)$ mode around $e \approx 0.3$. In these cases, the inverse iteration procedure converges toward an eigenmode corresponding to a much higher $n$ value, whatever the starting point may be, at least in our range of investigation. It is worth noting that this procedure is indeed more efficient for well-separated eigenvalues, that is precisely not the case here. If the viscous decay rates are considered (see figure $4 \mathrm{~b}$ ), it is obvious that they do 
not follow a simple $e^{2}$ law, but exhibit anomalies in a way reminiscent of the behaviour of the spin-over mode, or even seem to diverge, such as the $(14,1,9)$ mode for $e>0.4$.

The spatial distribution has been also obtained, as shown in figures 7-9 for the viscous $(8,1,5),(14,1,9)$ and $(20,1,13)$ modes, for 3 values of eccentricity. Surprisingly, the cell-like features seen in the corresponding non-viscous modes are less apparent in the presence of viscosity, where they are replaced by geometrical structures more reminiscent of shear layers. These features makes also more difficult the identification of the modes found by the inverse iteration procedure. Resonance phenomena seem then to be present for these modes as for the spin-over one, but because several modes are involved in a limited eccentricity range, it may be difficult to associate precisely each anomaly with a given frequency crossing. To conclude, the proximity of several inertial modes lead them to enter into a resonance between each other through the viscosity, a behaviour more complex than expected in a simple, non-viscous approach. As well, it requires to go beyond the first-order perturbation approach followed in the literature (Greenspan, 1968; Zhang et al., 2004) to take these inter-mode resonances into account.

\subsection{Viscosity dependence of decay rates}

In order to better characterize how the decay rates are influenced by the viscosity, the $(2,1,1)$ and $(8,1,5)$ eigenmodes have been investigated by the inverse iteration method for several Ekman numbers (see figure 10). It appears that the anomalies occuring at the critical eccentricity are smoother and smoother as $E$ increases, a behaviour characteristic of resonance phenomena. For a value of about $10^{-4}$, they are almost completely damped and the theoretical variation is recovered. It is worth noting that the proximity of eigenfrequencies means also the proximity of boundary layer eruptions occuring at a critical latitude (see figure 5). It can be anticipated that these eruptions will play a crucial role in the viscous coupling between the corresponding modes, and it can be expected that the amplitude of this coupling will depend on both the separation of the critical latitudes and the viscosity. 


\section{Discussion}

In the present work, a detailed study of a few viscous $m=1$ eigenmodes of a rotating fluid within a spheroidal container has been performed by direct numerical simulation. In a linear approximation, their behaviour, namely their viscous decay rate, the viscous correction to their eigenfrequency and their spatial structure, has been investigated as a function of the eccentricity $e$ of the spheroidal container, for a Ekman number $E=10^{-5}$. A particular attention has been paid to the spin-over mode, which plays a fundamental role in the precession problem. As a main result, a strong slowing-down of its viscous decay rate $(\sim 10 \%)$ has been evidenced in the range $e \approx 0.5-0.6$, shown by a large anomaly of its eccentricity dependence. This can be considered as the signature of a resonance between several viscous eigenmodes, in an eccentricity range where their frequencies become very close to each other. This resonance has not been predicted in previous linear theory (Greenspan, 1968; Zhang et al., 2004). A higher order perturbation analysis is needed to account for such an effect, by explicitely considering the coupling between different eigenmodes propagating at frequencies close to each other. However, the eigenfrequency spectrum is known to be dense, so that there exists always many eigenmodes with a frequency close to that of a given mode. It can be assumed that a resonance between these modes should be renormalized by a kind of coupling constant or interaction integral, which could be large only for a reduced number of pair of modes. The magnitude itself of the decay rates, or the proximity of their value is probably involved also in the resonance phenomenon. A further analysis is required to elucidate this point. As well, the consequences of such a resonance on possible fluid instabilities remain to be investigated. Moreover, previous studies in spherical geometry have shown how the spatial structure of the spin-over mode changes as a function of the viscosity, i.e. how the internal shear layers scale with the Ekman number (Hollerbach \& Kerswell, 1995; Noir et al., 2001). One may wonder whether the same $E^{1 / 5}$ scaling apply to the present case with a large eccentricity, where the shear 
layers have been replaced by cell-like structures, at least for the $E$ value used in the present calculation.

\section{Acknowledgments :}

Most of the computations presented here have been performed at the Institut $d u$ Développement et des Ressources en Informatique Scientifique (IDRIS), under the project 41712 . 


\section{FIGURE CAPTIONS}

Fig. 1: Calculated variation of renormalized decay factor $\square \cdot E^{\square 1 / 2}$ (left scale) and eigenfrequency $\square_{d}$ (right scale) of the spin-over mode for eccentricities $e=0.02$ (left) and 0.51 (right) and for a Ekman number $E=10^{-5}$.

Fig. 2: Eccentricity dependence of the renormalized decay factor $\square E^{\square / 2}$ (left) and of $\square_{0}$ (middle) and $\square_{1}$ (right) contributions to the eigenfrequency $\square_{d}$ for the spin-over mode; continuous lines are theoretical predictions; dashed lines correspond to the present timestepping simulations, dotted lines to inverse iteration results; arrows indicate the crossing between the spin-over frequency and the $(8,1,5)$ and $(14,1,9)$ frequencies, respectively.

Fig. 3: The three components of the residual fluid velocity of the spin-over mode within the meridional plane containing the $\ell$ vector, for different eccentricities, after removing the inviscid contribution (see text); velocities are normalized so that $|\mathbf{u}|_{\max }=1$; note the spatial evolution of the azimutal component.

Fig. 4: (a): Eccentricity dependence of eigenfrequencies, for some inviscid (lines) and viscous (symbols) spheroidal inertial modes close to the $(2,1,1)$ spin-over mode; (b): Eccentricity dependence of corresponding decay rates. Continuous line and full circles: $(2,1,1)$ mode; dashed line and open squares: $(8,1,5)$ mode; dotted line and open circles: $(14,1,9)$ mode; triangles: $(20,1,13)$ mode; lines are theoretical predictions.

Fig. 5: The three velocity components of the $(8,1,5),(14,1,9)$ and $(20,1,13)$ inviscid inertial modes for $e=0.39$; the critical latitude for viscous boundary layer eruptions is indicated by a star on the spheroidal boundary.

Fig. 6:The three components of the residual fluid velocity of the viscous spin-over mode within the meridional plane containing the $\ell$ vector (top) and perpendicular to it (bottom), for $e=0.39$. 
Fig. 7:The three velocity components of the $(8,1,5)$ (top), $(14,1,9)$ (middle) and $(20,1,13)$ (bottom) viscous inertial modes for $e=0.02$; velocities are normalized so that $|\mathbf{u}|_{\max }=1$.

Fig. 8: The three velocity components of the $(8,1,5)$ (top), $(14,1,9)$ (middle) and $(20,1,13)$ (bottom) viscous inertial modes for $e=0.27$; velocities are normalized so that $|\mathbf{u}|_{\max }=1$.

Fig. 9: The three velocity components of the $(8,1,5)$ (top), $(14,1,9)$ (middle) and $(20,1,13)$ (bottom) viscous inertial modes for $e=0.39$; velocities are normalized so that $|\mathbf{u}|_{\max }=1$.

Fig. 10: Eccentricity dependence of the renormalized decay factor $\square E^{\square 1 / 2}$ for (a): the $(2,1,1)$ and $(b)$ : the $(8,1,5)$ modes, for various Ekman numbers ranging from $10^{-4}$ to $10^{-5.25}$; continuous lines are theoretical predictions. 


\section{REFERENCES}

BACKUS, G., PARKER, R. \& CONSTABle, C. 1996 Foundations of Geomagnetism. Foundations of Geomagnetism. Cambridge University Press, Cambridge.

BUSSE, F. H. 1968 Steady fluid flow in a precessing spheroidal shell. J. Fluid Mech. 33, 739-751.

GREENSPAN, H. P. (Editor), 1968. The theory of rotating fluids. The theory of rotating fluids. Cambridge University Press, Cambridge.

HOLLERBACH, R. \& KERSWELL, R. R. 1995 Oscillatory internal shear layers in rotating and precessing flows. J. Fluid Mech. 298, 327-339.

KERSWELL, R. R. 1993 The instability of precessing flow. Geophysical Astrophysical Fluid Dynamics 72, 107-144.

KERSWELL, R. R. 1994 Tidal excitation of hydromagnetic waves and their damping in the Earth. J. Fluid Mech. 274, 219-241.

LIAO, X., ZHANG, K. \& EARNSHAW, P. 2001 On the viscous damping of inertial oscillation in planetary fluid interiors. Phys. Earth Planet. Inter. 128, 125-36.

LORENZANI, S. \& TILGNER, A. 2001 Fluid instabilities in precessing spheroidal cavities. J. Fluid Mech. 447, 111-128.

LORENZANI, S. \& TILGNER, A. 2003 Inertial instabilities of fluid flow in precessing spheroidal shells. J. Fluid Mech. 492, 363-79.

NOIR, J., JAULT, D. \& CARDIN, P. 2001 Numerical study of the motions within a slowly precessing sphere at low Ekman number. J. Fluid Mech. 437, 283-299.

POINCARE, R. 1910 Sur la précession des corps déformables. Bulletin Astronomique 27, 321-356.

RIEUTORD, M. \& VALDARETTO, L. 1997 Inertial waves in a rotating spherical shell. $J$. Fluid Mech. 341, 77-99.

SCHMITT, D. \& JAULT, D. 2004 Numerical study of a rotating fluid in a spheroidal container. J. Comput. Phys. 197, 671-85.

STEWARTSON, K. \& ROBERTS, P. H. 1963 On the motion of a liquid in a spheroidal cavity of a precessing rigid body. J. Fluid Mech. 17, 1-20.

StOER, J. \& BULIRSCH, R. 1980 Introduction to Numerical Analysis. Springer, NewYork.

TILGNER, A. 1999 Non-axisymmetric shear layers in precessing fluid ellipsoidal shells. Geophys. J. Int. 136, 629-36.

TILGNER, A. \& BUSSE, F. H. 2001 Fluid flows in precessing spherical shells. J. Fluid Mech. 426, 387-396.

WALKER, M. R. \& BARENGHI, C. F. 1994 High resolution numerical dynamos in the limit of a thin disk galaxy. Geophysical and Astrophysical Fluid Dynamics 76, 265.

ZHANG, K., LIAO, X. \& EARNSHAW, P. 2004 On inertial waves and oscillations in a rapidly rotating spheroid. J. Fluid Mech. 504, 1-40. 

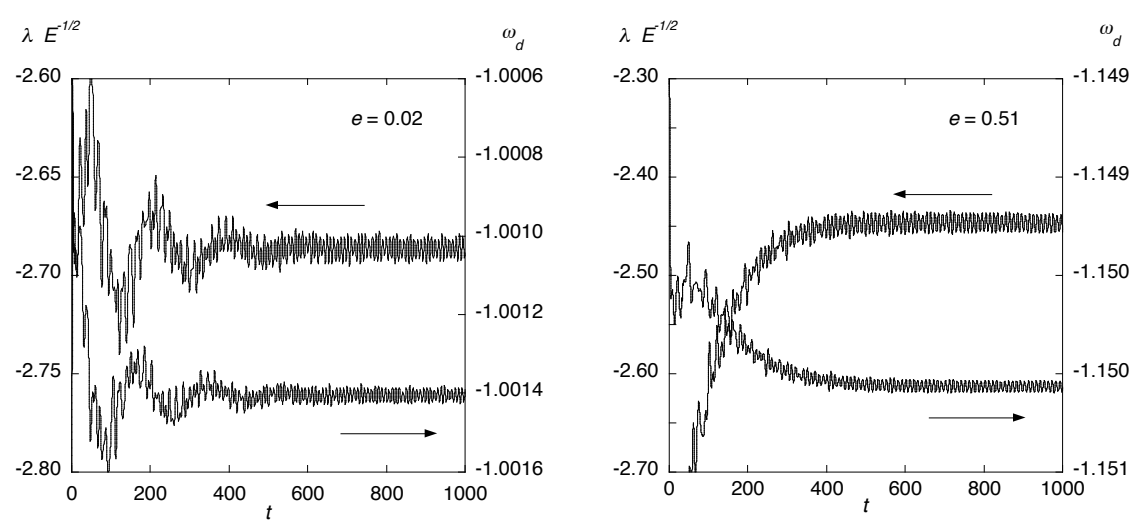


\section{Page 21 of 29}
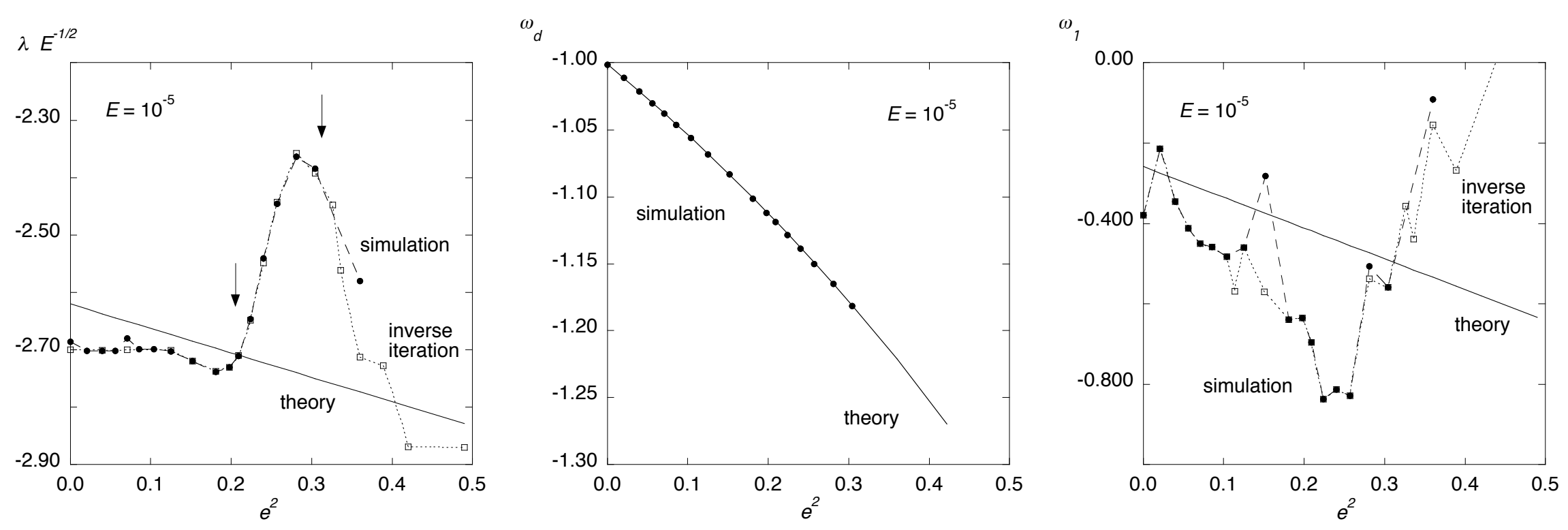

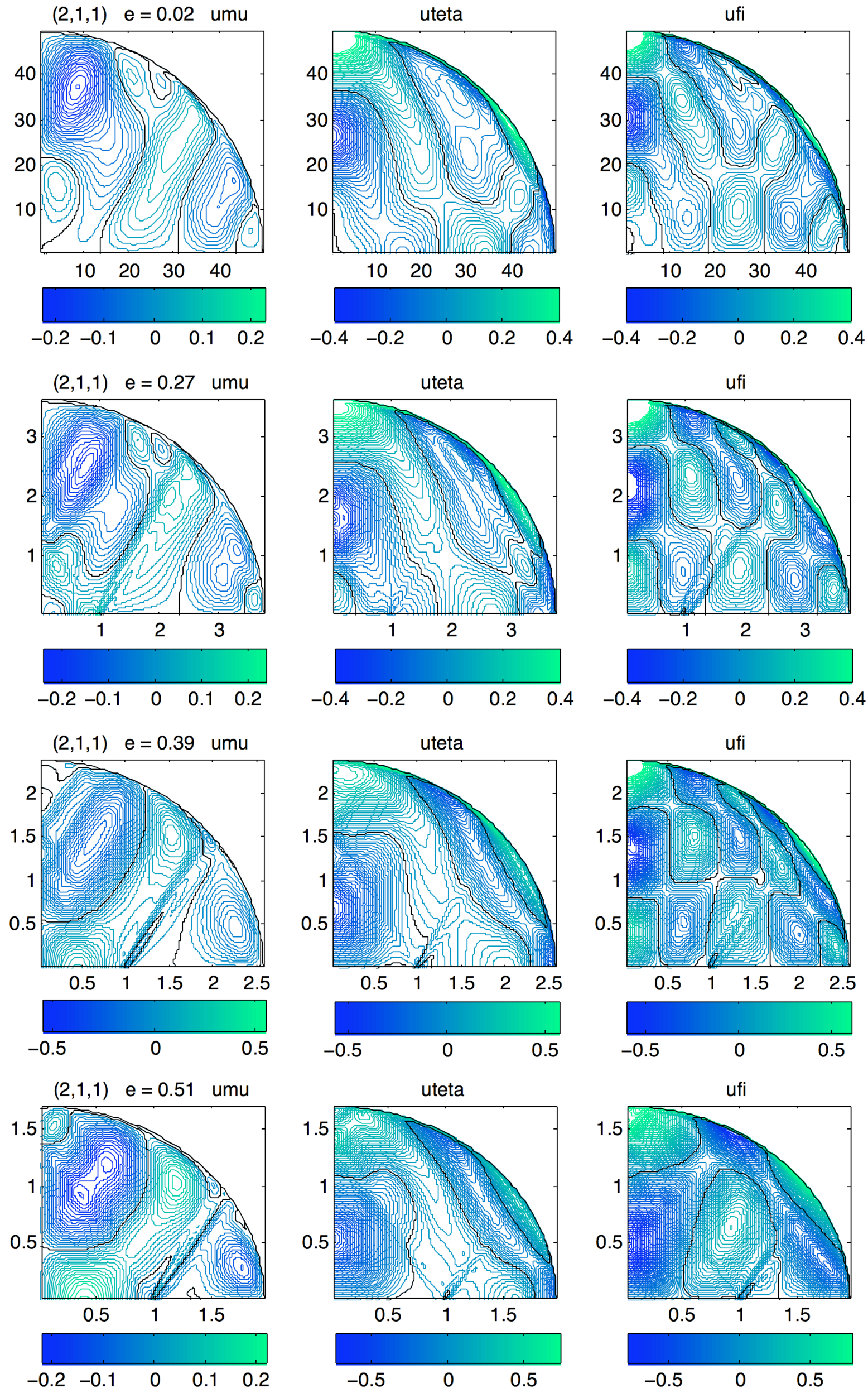


\section{Page 23 of 29}
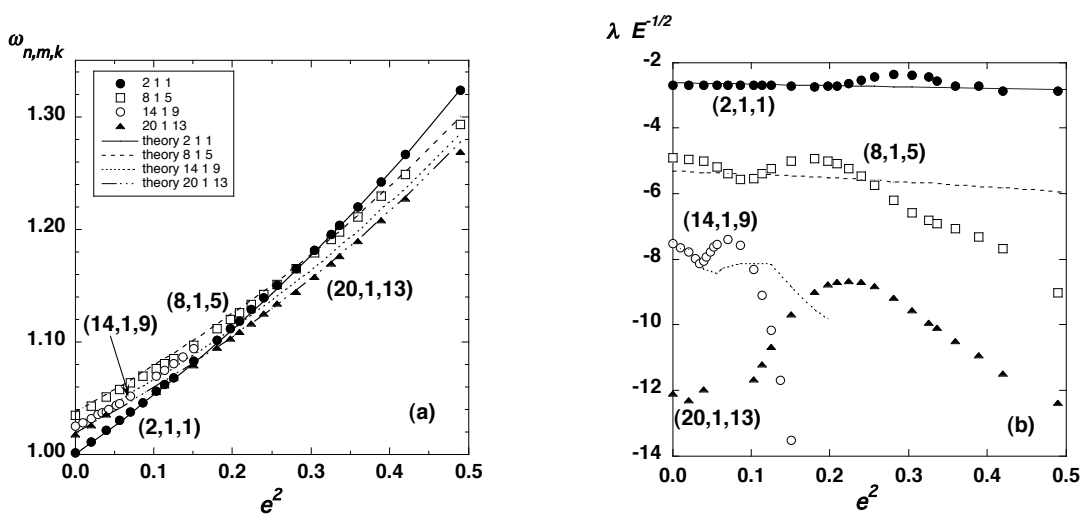

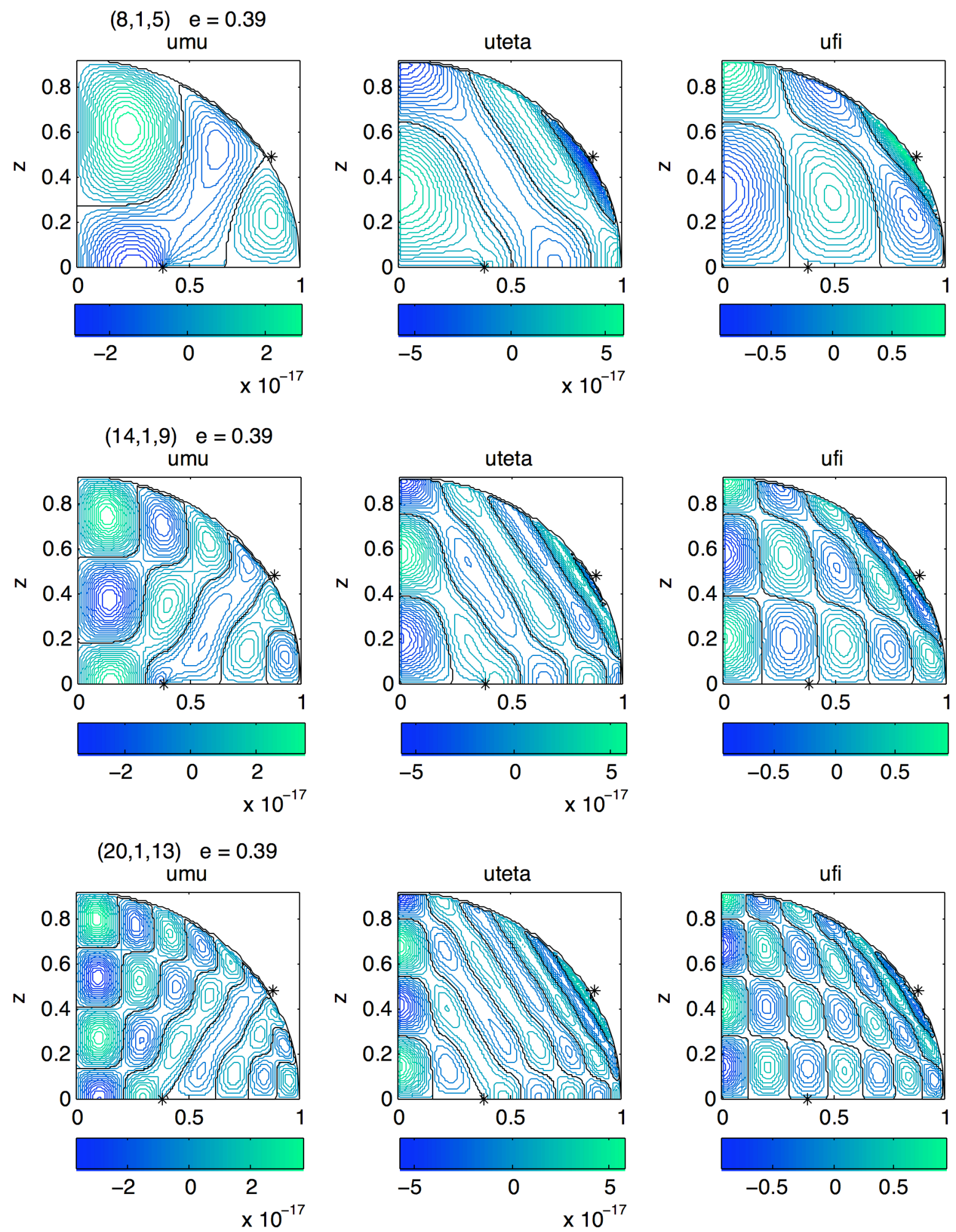

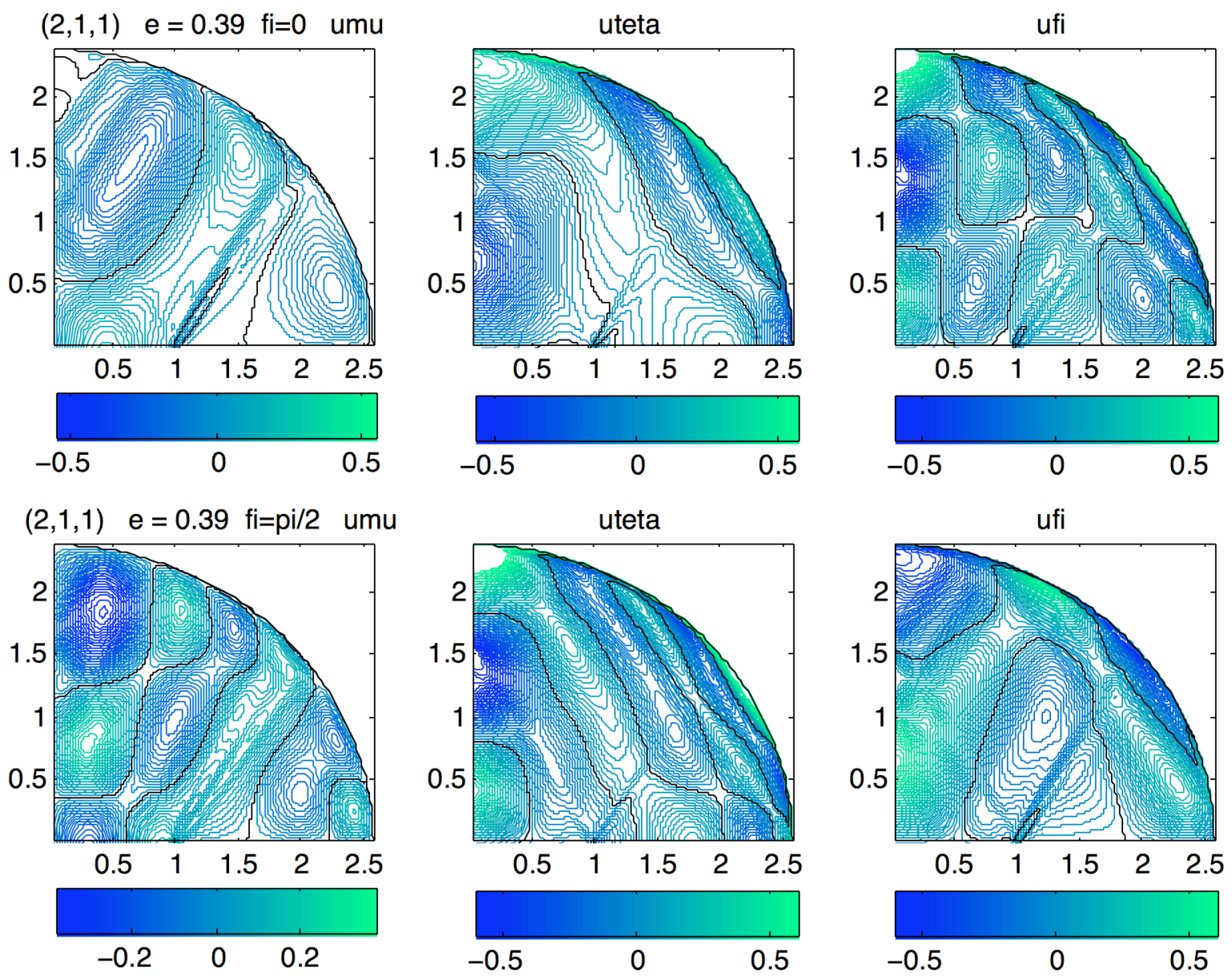

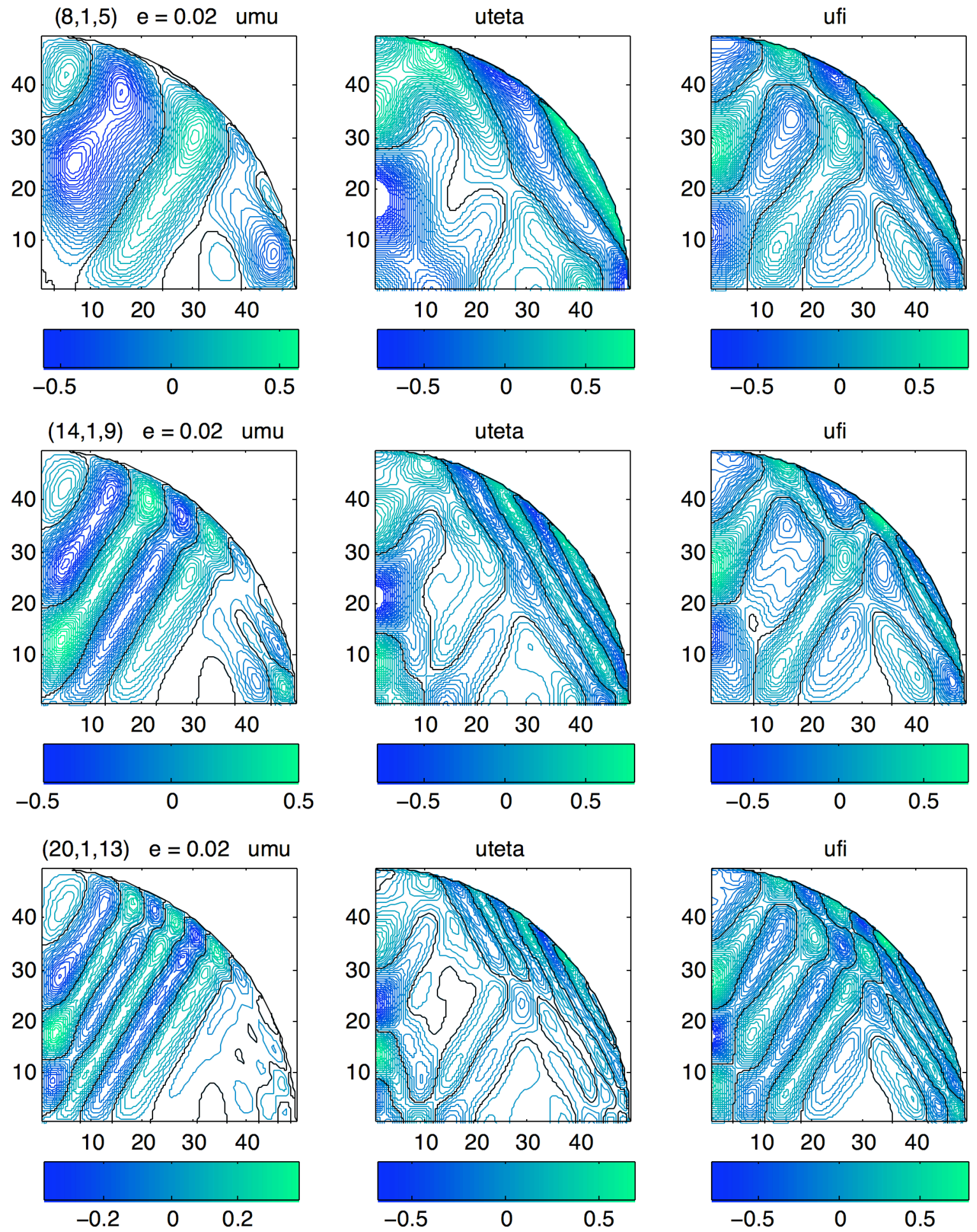

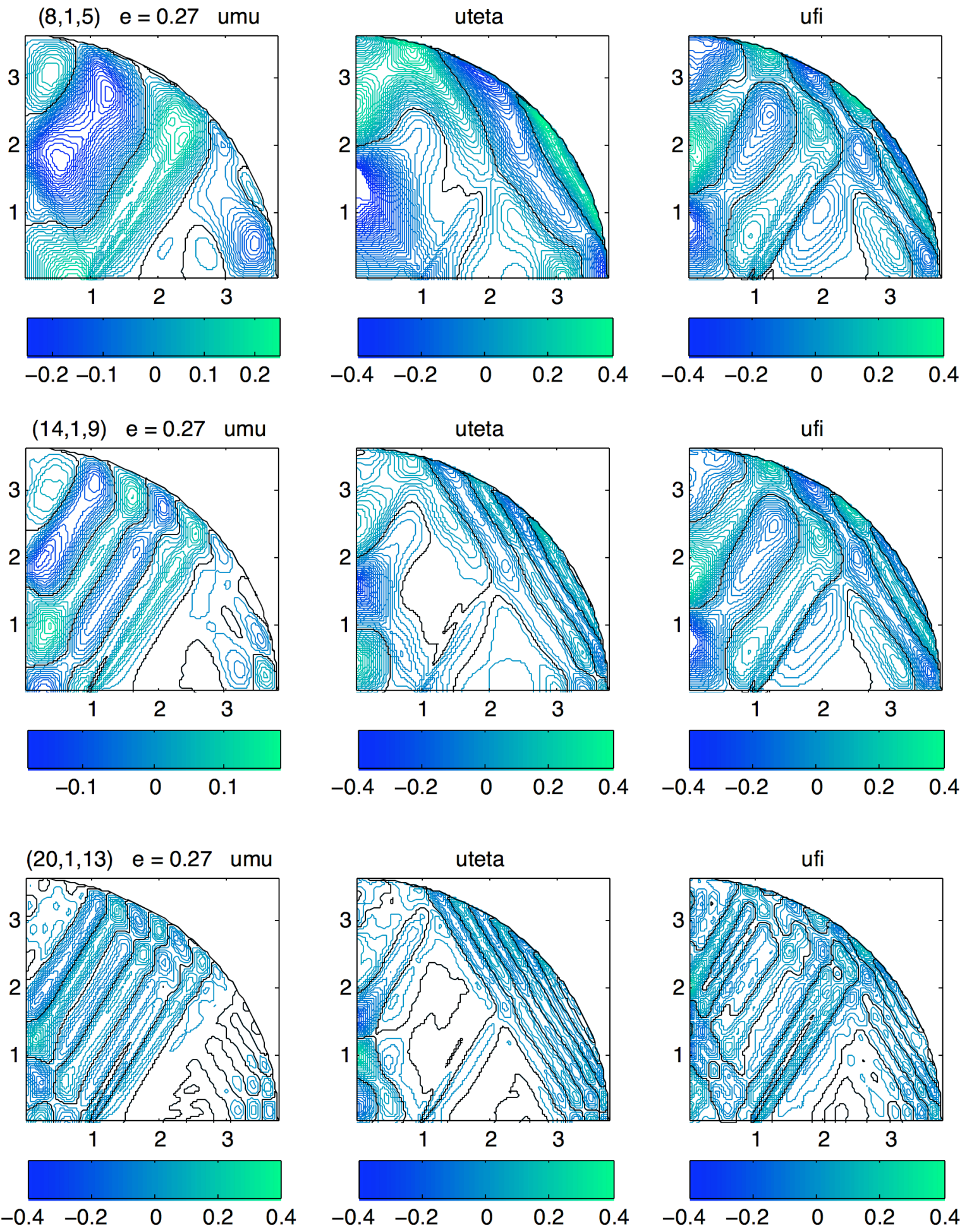

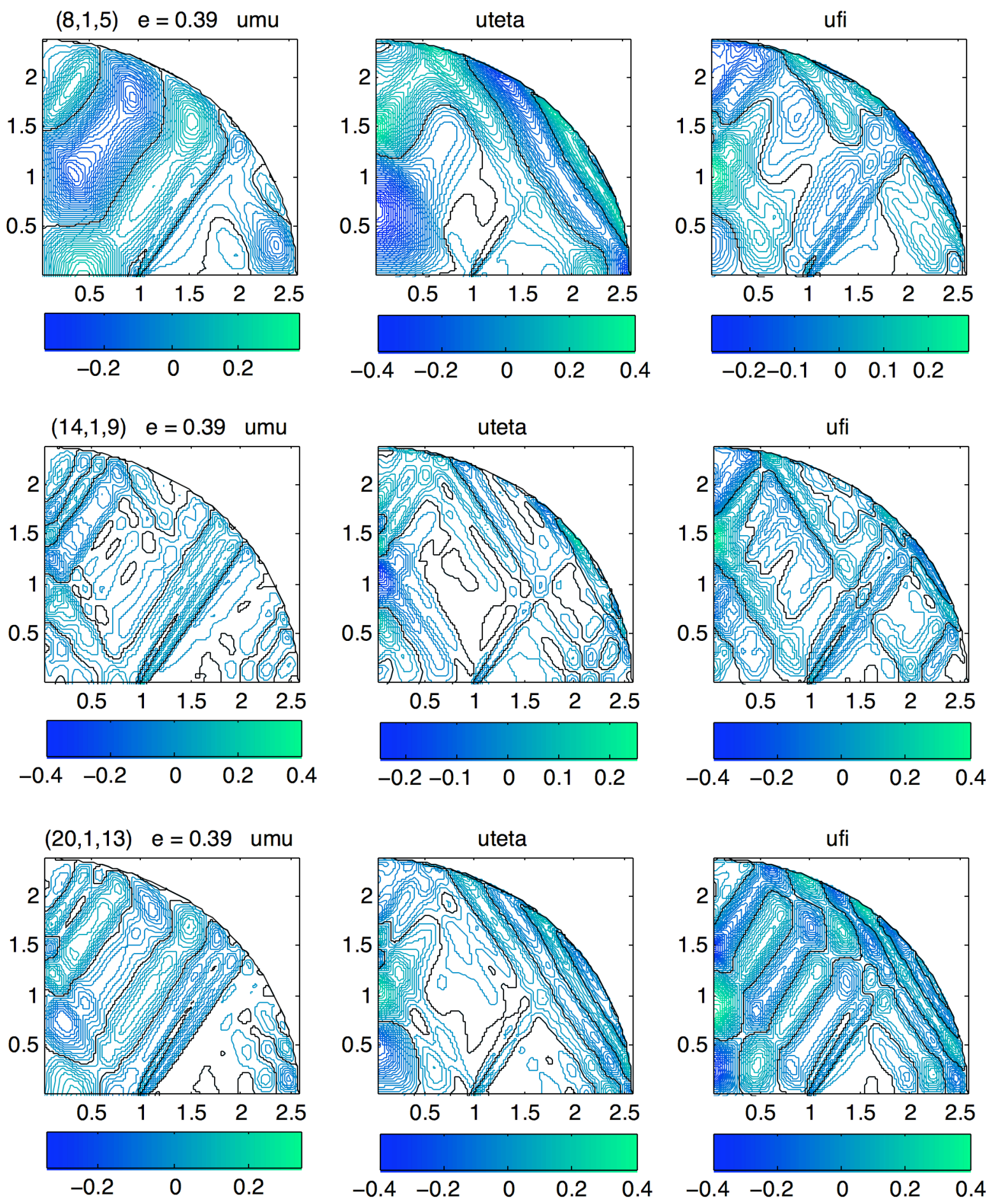

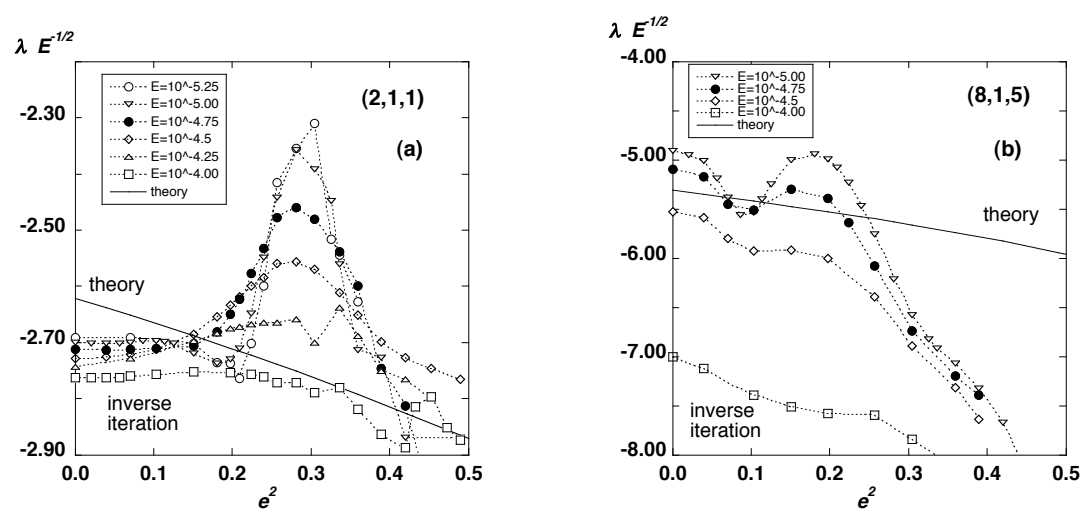